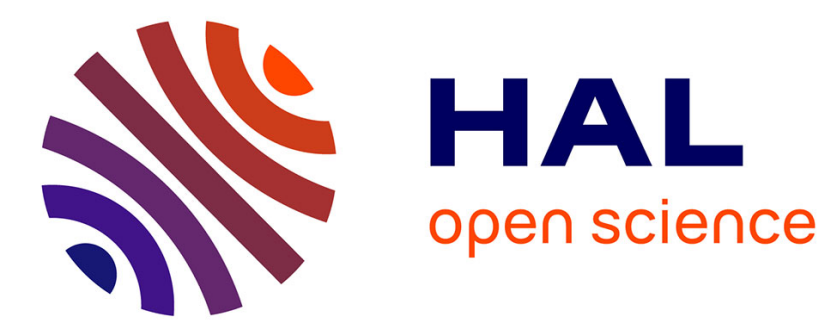

\title{
Tokamak discharge simulation coupling freeboundary equilibrium and plasma model with application to JT-60SA
}

\author{
V. Ostuni, Jean François Artaud, G. Giruzzi, Emmanuel Joffrin, Holger \\ Heumann, H. Urano
}

\section{To cite this version:}

V. Ostuni, Jean François Artaud, G. Giruzzi, Emmanuel Joffrin, Holger Heumann, et al.. Tokamak discharge simulation coupling freeboundary equilibrium and plasma model with application to JT60SA. Nuclear Fusion, 2021, 61, pp.026021. 10.1088/1741-4326/abcdb7 . cea-03287071

\section{HAL Id: cea-03287071 https://hal-cea.archives-ouvertes.fr/cea-03287071}

Submitted on 15 Jul 2021

HAL is a multi-disciplinary open access archive for the deposit and dissemination of scientific research documents, whether they are published or not. The documents may come from teaching and research institutions in France or abroad, or from public or private research centers.
L'archive ouverte pluridisciplinaire HAL, est destinée au dépôt et à la diffusion de documents scientifiques de niveau recherche, publiés ou non, émanant des établissements d'enseignement et de recherche français ou étrangers, des laboratoires publics ou privés. 


\title{
Tokamak discharge simulation coupling free- boundary equilibrium and plasma model with application to JT-60SA
}

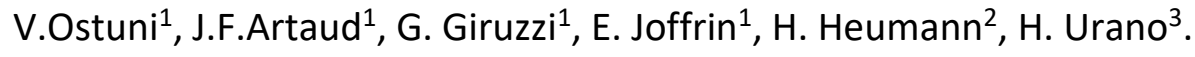 \\ ${ }^{1}$ CEA, IRFM, F-13108 Saint-Paul-lez-Durance, France. \\ ${ }^{2}$ Université Nice Sophia Antipolis, Laboratoire J.A. Dieudonné CNRS UMR 7351, INRIA CASTOR \\ Team-Project, Parc Valrose, 06108 Nice Cedex 02, France \\ ${ }^{3}$ National Institutes for Quantum and Radiological Science and Technology, Naka, Ibaraki, 311-0193, Japan \\ E-mail contact of main author: valeria.ostuni@cea.fr
}

\begin{abstract}
In order to simulate a full tokamak discharge, a fast integrated tokamak modelling tool for scenario design, METIS, is coupled with a quasi-static free-boundary magnetic equilibrium code, FEEQS. The first code is able to compute at each time the plasma equilibrium, the current density and plasma pressure profiles. The generated outputs are iteratively used by the second code that computes the poloidal field coils currents needed in order to obtain a given plasma shape. This is used to assess the feasibility of scenarios designed by integrated modelling simulations. This approach is much faster with respect to full simulators, equipped with specific feedback controllers, and can be regarded as complementary or preparatory to much more timeconsuming scenario control optimisation. The results obtained with the equilibrium code are benchmarked with data already available and computed using the TOSCA code. To illustrate how the coupled codes work, the optimization of one of the reference scenarios of the JT-60SA tokamak is carried out. The use of this new simulation tool for tokamak scenario design is discussed.
\end{abstract}




\section{INTRODUCTION}

The development and qualification of tokamak operating scenarios have crucial importance in achieving the objectives of the ITER project and in defining the plasma scenario design for DEMO, the first demonstration fusion reactor. Combining experiments and theory-based models, a set of coherent, machine-independent and reproducible plasma properties can be defined for each scenario. Predicted simulations not only mitigate the risks of ITER operation but can also reduce time, cost and resources to extrapolate useful results from the available data to the next generation facilities. For this reason, integrated modelling of burning plasmas has to be as accurate as possible and it has to take into account plasma core and edge physics as well as the control schemes, the plasma-materials interaction and the mechanical and electromagnetic limits of the tokamak components. The development of a reliable and validated computer code having as input the same control parameters used to pilot the machine and able to predict the full-time evolution of a tokamak discharge is a high-level objective of fusion research. [1]

The preparation and analysis of a tokamak discharge can be carried out by the use of tokamak simulators that include not only the plasma description but also all or some parts of the plant systems. This kind of simulator can help to answer the two main questions, i.e., feasibility and controllability of the plasma discharge. The main constraints on feasibility or controllability in tokamak are imposed not only by the physics properties of the plasma, but mainly by the magnet system and in particular, the poloidal system coils and the associated power supply. In order to address these two questions, simulators coupling plasma models, free-boundary equilibrium codes, power supply models and control systems (kinetic and magnetic) have been developed and used. As relevant examples, various codes can be mentioned: DINA [2] , CORSICA [ $\underline{3}$ ], DINA-CH coupled to the CRONOS suite of codes [4], ASTRA coupled to SPIDER [ $\underline{5}$ ], JINTRAC coupled to CREATE-NL [ 6 ]. These simulations are quite demanding in terms of computing time and fine-tuning of input parameters, but they allow answering both questions of feasibility and controllability of the discharge. Nevertheless, to explore and prepare experiments, it is interesting to have faster and simpler to manage simulators that answer only part of the questions. This fast simulator can be used beforehand the full simulator, to prepare and greatly simplify its task, or for testing scenario properties during the experiments.

We have built a simulator of this kind by coupling the fast integrated modelling code METIS [7] with the free boundary equilibrium code FEEQS [8] and applied it to the study of one of the reference JT-60SA scenarios. The principle of this simulator is to first design the plasma scenario by METIS and then compute the currents in the poloidal field coils needed to obtain that plasma by using the code FEEQS in inverse mode. In contrast with a full simulator, this combination allows faster computation as the time step can be arbitrarily chosen: only selected time slices of the plasma simulation are computed with the equilibrium code and no convergence loop has to be done. In return, we answer only part of the questions asked. The outputs of FEEQS code are currents in the coils, voltages at coil terminals, maximum forces on the coils, maximum magnetic field on coils conductors and last closed flux surface (LCFS) curve which is solution of the equilibrium equation. With these data, we are able to assess the feasibility of the discharge, but not its controllability. To answer the question of feasibility, we check if currents, voltages, forces, magnetic fields are inside the limits imposed by machine and power supply design and if the LCFS output of FEEQS is close to the one used in the METIS simulation. The scenario can then be optimized by iterating the runs of METIS and FEEQS, updating the LCFS curves.

Once we are confident in the feasibility of the discharge, the next step is to check the controllability with the full simulator. Currently, a dedicated simulator based on CREATE-NL [ $\underline{9}$ ] and METIS is being 
developed for JT-60SA studies. As for the full simulation we start from a scenario that is already optimised by the fast simulator, the full simulation will be simpler to realise. Additionally coil current waveforms provided by FEEQS code can be directly used as feedforward waveforms for the full simulator.

The tokamak JT-60SA [10] was chosen as a first application to test the coupled codes. JT-60SA will support the ITER experimental program realizing stable steady-state operations and it will provide key information for the design of DEMO scenarios that should be operated in a higher normalized beta regime. In order to satisfy all of the central research needs for ITER, three main scenarios (corresponding to the main scenarios of ITER) will be tested during the operation phases: the standard H-mode operation scenario, the advanced inductive (hybrid) operation scenario and steady-state operation scenario [11].

The results obtained running the coupled codes for the JT-60SA H-mode scenario are compared with data already available and computed using other codes. Once this validation step accomplished, the optimization of one of the scenarios is carried out to show an example of how the two codes work.

In this paper, the main characteristics of the two coupled codes for the scenario simulation are reported and explained separately in section 2; section 3 reports the benchmark and the coupling procedure of them; section 4 concerns the results obtained using the two codes in order to optimize the JT-60SA H-mode scenario. Conclusions are given in section 5.

\section{CODES FOR SCENARIO SIMULATION}

A code able to integrate transport equation with magnetic equilibria is not sufficient to simulate a whole tokamak discharge. In addition, it is necessary to compute the time series of currents applied into the poloidal field coils. For this reason, a fast integrated modelling code (METIS) has been coupled with a free-boundary equilibrium evolution code (FEEQS) in order to work together as a fast discharge simulator. In this section, the main elements of the two codes used in the coupling are shortly described, since details of both codes can be found in specific references [7,8].

\subsection{METIS code}

METIS [7] is a fast integrated modelling code that allows building a realistic simulation of plasma scenarios in about one-minute computation time on a single node, even for full ITER discharges of 1000s duration. Originally, it was built as a module of the integrated modelling suite of codes CRONOS [12], later it has been developed as an autonomous code. In order to be faster than a 1.5D code (2D equilibria and 1D transport equation) and to keep the reliability of the simulation results, specific developments were necessary to establish what are the aspects of the problem that can be modelled in a simpler way. The current diffusion and plasma equilibrium equations are kept in a 1.5D scheme, as in CRONOS. The first

one is solved in terms of poloidal flux using a toroidal flux coordinate $\rho$ grid, with $\rho=\sqrt{\frac{\Phi}{\pi B_{0}}}$ where $\Phi$ is the flux of the toroidal magnetic field and $B_{0}$ the vacuum toroidal field at the center of the vacuum vessel. The radial grid is equally spaced in normalized toroidal flux coordinate $\rho_{\text {norm }}=\rho / \rho \mathrm{m}$. The equation solved is:

$$
\left.\frac{\partial \Psi}{\partial t}\right|_{\rho_{\text {norm }}}=\frac{\left\langle\frac{|\nabla \rho|^{2}}{R^{2}}\right\rangle}{\mu_{0} \sigma_{\|} \rho_{m}^{2}\left\langle\frac{1}{R^{2}}\right.} \frac{\partial^{2} \Psi}{\partial \rho_{\text {norm }}^{2}}+\left\{\frac{\left\langle\frac{|\nabla \rho|^{2}}{R^{2}}\right\rangle}{\mu_{0} \sigma_{\|} \rho_{m}^{2}\left\langle\frac{1}{R^{2}}\right\rangle} \frac{\partial}{\partial \rho_{\text {norm }}}\left[\ln \frac{\left.\frac{\partial V}{\frac{\partial \rho \text { norm }}{F}} \frac{|\nabla \rho|^{2}}{R^{2}}\right\rangle}{F}\right]+\frac{\rho_{\text {norm }}}{\rho_{m}} \frac{d \rho_{m}}{d t}+\frac{\rho_{\text {norm }}}{2 B_{0}} \frac{d B_{0}}{d t}\right\} \frac{\partial \Psi}{\partial \rho_{\text {norm }}}+\frac{B_{0}}{\left.\sigma_{\| F} F \frac{1}{R^{2}}\right\rangle} j_{n i}
$$


Where $\left.\frac{\partial \Psi}{\partial t}\right|_{\rho_{\text {norm }}}$ is the time derivative of the poloidal flux at a given radial position $\rho_{\text {norm }}, \sigma_{\mathrm{V}}$ is the parallel electrical conductivity, $\mathrm{F}$ is the diamagnetic function ( $F=R B_{\phi}$ with $B_{\phi}$ the toroidal component of the magnetic field) , $j_{n i}$ is the current density driven by non-inductive sources, $\mathrm{R}$ the major radius, $\mu_{0}$ is the magnetic permeability of free space and $\rho_{m}$ the value of $\rho$ at the last closed flux surface (LCFS). The notation \langle\rangle indicates a magnetic flux surface average, defined as the volume average of a quantity around a flux surface of radial coordinate $\rho$. Equation (1), already used and discussed in $[7,12]$ is the customary current diffusion equation, only cast in such a way that poloidal flux is the unknown quantity and the normalized toroidal flux coordinate is the spatial coordinate. Note that, because of this choices, it contains terms related to geometry transformations (such as $\left.\left\langle\frac{|\nabla \rho|^{2}}{R^{2}}\right\rangle\right)$. Moreover, it contains terms describing the time evolution of the LCFS (via $\rho_{m}$ ) and confining magnetic field $B_{0}$, in order to fully describe the flux evolution during the discharge.

In order to speed up the calculation, the current diffusion is computed on a 21 points radial grid only. The modelling of heat transport is the part of the code for which several different models exist and consuming the largest CPU time; therefore, it is treated as a mixed OD-1D approach to save computation time. The first step consists of solving the equation for the time evolution of the plasma thermal energy content, the second step consists of computing the electron and ion temperatures profiles solving the stationary transport equation at selected time slices. The electron density profile in L-mode is computed starting from the prescription of three parameters that are: the line-averaged density $\bar{n}_{e}$, the peaking factor $\frac{n_{0}}{\langle n\rangle}$ and the density value at the separatrix $n_{e, a}$. In H-mode, the density at the top of pedestal (always at $\frac{r}{a}=0.95$ in METIS) is also constrained by a specific scaling law. All of the other species density profiles are assumed proportional to the electron density, except for the tungsten [7]. In addition, an estimate of plasma rotation is computed taking into account the effect of neutral beam torque and intrinsic rotation. The model consists in evaluate the volume averaged toroidal momentum, the rotation at the LCFS and, in the end, the radial profiles of toroidal and poloidal rotation velocities and the radial electric field (details are available in reference [7]).

METIS includes fast solvers for the computation of the source terms for the heat transport equation and the current diffusion one. The source models are chosen in order to be fast and simple avoiding increase of computational speed. The sources taken into account are NBI (Neutral Beam Injection), ICRH (Ion Cyclotron Resonance Heating), LHCD (Lower Hybrid Current Drive), ECRH (Electron Cyclotron Resonance Heating), pellets injection, fusion reaction of thermal ions, ripple effects, wall recycling and radiative processes.

METIS can use a description of plasma geometry given either by moments of the magnetic equilibrium shape (Shafranov shift, ellipticity and triangularity) or by a list of points describing the LCFS. In the latter case, the list of $(R, Z)_{K}$ points are used to recompute the moments and the global geometric quantities. In this case, the equilibrium mesh is corrected using a morphing function, describing the transition from the shape of the LCFS to that of the core flux surfaces, in such a way that the current diffusion equation is solved using geometrical coefficients coherent with the actual flux surface shape [7].

A predictive simulation of the $\mathrm{H}$-mode reference scenario of JT-60SA (so called scenario 2 of Ref. [11]) is done using METIS [13]. This scenario is characterized by a plasma current of $5.5 \mathrm{MA}$, a toroidal magnetic field of 2.25 T, a total heating power of $41 \mathrm{MW}$ (10 MW of Negative lon based NBI, $24 \mathrm{MW}$ of Positive lon based $\mathrm{NBI}$ and $7 \mathrm{MW}$ of $\mathrm{ECRH}$ ) and a safety factor at $95 \%$ of the poloidal flux $\mathrm{q}_{95}=3$. For the parameters of this reference scenario, the radial position of the ECRH power deposition is varied to illustrate an example of how METIS works (see Figure 1(a)). Figure 1(b) and Figure 1(c) show the impact of the variation of the radial deposition on the total current density profiles and on the electron temperature profiles. The radial shift of the deposition location leads to a shift of the current density peak in the radial coordinate, 
since a significant fraction of the current is non-inductively driven by the EC waves, as well as a marked change of the electron temperature profile peaking.
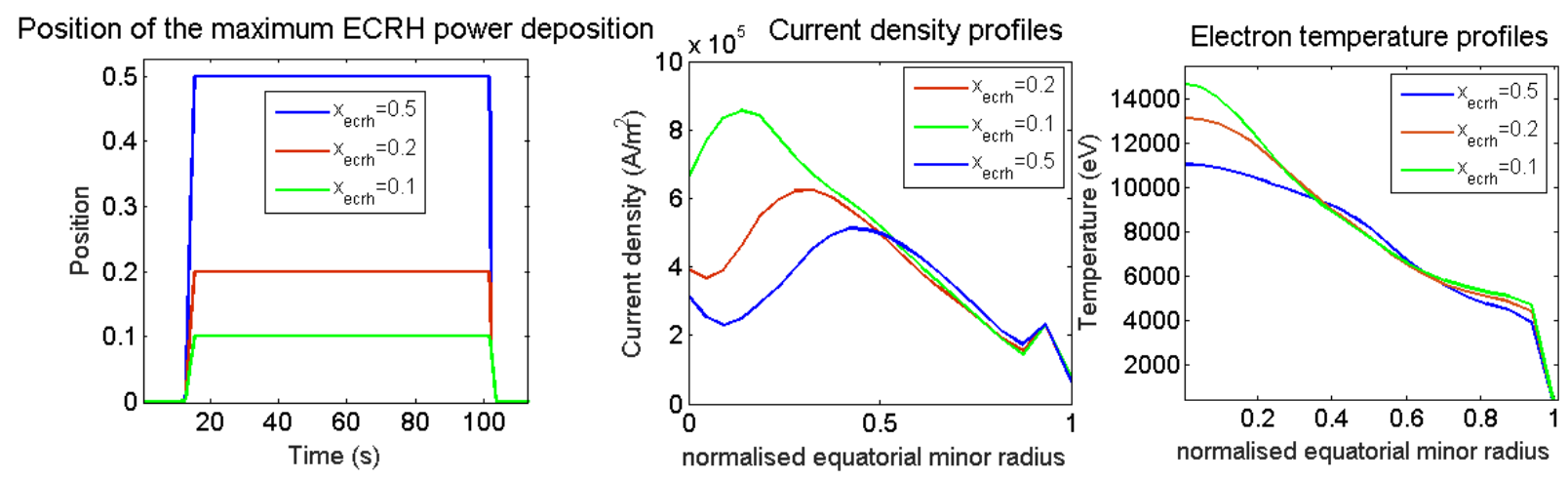

Figure 1. (a) ECRH radial power deposition positions used as input parameters in METIS for three different simulations of the standard H-mode operation scenario of JT-60SA. (b) Impact of the variation of the radial deposition on the total current density profiles computed by METIS. (c) Impact of the variation of the radial deposition on the electron temperature profile computed by METIS. The abscissa is the normalized Lao coordinate (normalized equatorial minor radius) [14]

\subsection{FEEQS code}

FEEQS [8] is a free-boundary equilibrium code. It relies on an approximation of the magnetic flux based on a finite element method with triangular mesh and piecewise linear shape functions. Decaying conditions of the flux at infinity are coupled in via boundary integral equations [15]. Accurate linearization allows for fast converging Newton-type iteration schemes to solve the resulting non-linear discrete versions of the free-boundary equilibrium problems. Newton-type iterations avoid the severe convergence problems $[16,17]$ observed frequently with fixed-point iterations schemes for elongated plasma equilibria. As a result, FEEQS can perform the simulations of physically unstable equilibria without the support of a feedback-control system. This allows to decouple the design of discharge scenarios from the specific form of the controllers. The feedback-control system enters only in a final step, where a scenario is validated in combining feedback-controllers and a full simulator. Neither a specific feedbackcontrol system nor the final validation step is the focus of this contribution. Nevertheless, FEEQS has all the capabilities to double as a full simulator, as it provides functions that combine the classical GradShafranov equation for MHD equilibrium in an axisymmetric system with circuit and induction equations for poloidal field coils and passive structures inside the tokamak.

This contribution focuses on a different outstanding feature of FEEQS: its ability to solve so-called inverse equilibrium problems. For example, instead of finding an equilibrium for a given set of coil currents, it is possible to determine coil currents such that the plasma equilibrium has certain prescribed features. Such features may include, among others, the shape of the LCFS, the value of the flux at the boundary or technological limits such as force and current limits. These inverse problems are formulated as constrained optimization problems, where the usual plasma equilibrium equations are among the constraints and the desired features of an equilibrium are either enforced through additional constraints or penalization terms in the objective function. Here again, the accurate linearizations allow for fast converging iteration schemes (known as Sequential Quadratic Programming) to find solutions of the constrained optimization problems. 
FEEQS is written in MATLAB and hence, easy to use and extend. All core numerical computations are fully vectorized, which make the performance comparable to plain $\mathrm{C} / \mathrm{C}++$ implementations, but keeps a high degree of flexibility to be combined with other modules.

The outputs obtained from a METIS simulation are given as inputs in FEEQS. Typical results for the standard H-mode operation scenario of JT-60SA, computed using the inverse mode, are reported in Figure 2, where CS indicates the central solenoid coils, EF the equilibrium field coils, FPPC the fast plasma position control coils. Figure 2(a) shows the cross-section of the magnetic surface, as well as the values of each coil current computed at a given time and a given LCFS. Figure 2(b) shows the time behavior for the EF3 coil. The time evolution of several other quantities (defined as input or computed by METIS) are shown in Figures 2(c-g), in order to illustrate the characteristics of this scenario.
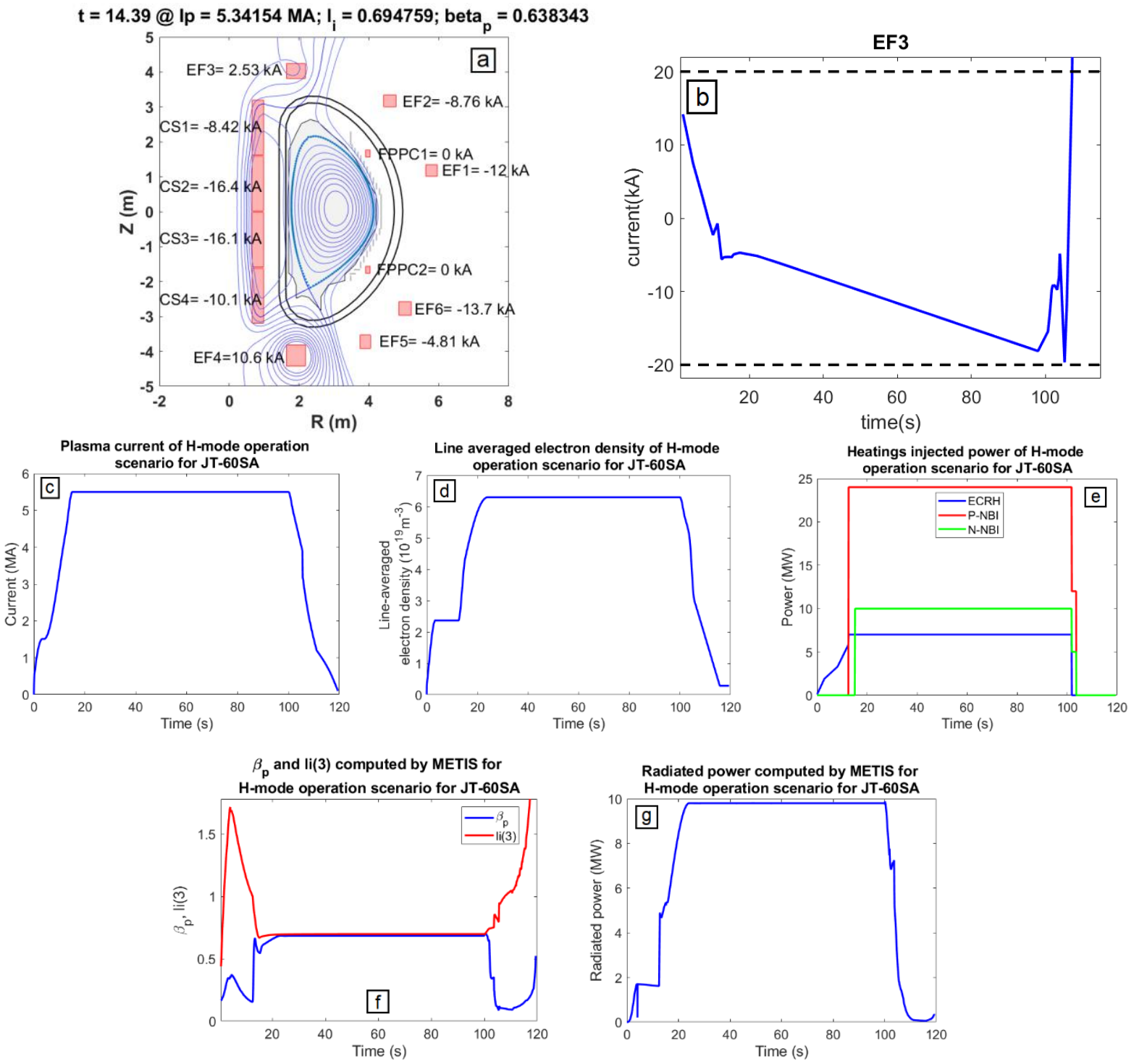

Figure 2 - FEEQS computation for the reference H-mode operation scenario of JT-60SA. (a) values of coils current computed by FEEQS for the time=14.39s and a given LCFS. Also, the value of the plasma current, li(3) and $\beta_{\mathrm{p}}$ are computed at time=14.39 s. Note: CS indicates the central solenoid coils, EF the equilibrium field coils, FPPC the fast plasma position control coils. (b) Time behavior of EF3 coil current. Dashed-lines correspond to the operational coils limit given to FEEQS as input parameters. (c-g): Time traces of the main parameters of $\mathrm{H}$-mode operation scenario for JT-60SA. Plasma current (c), line averaged electron density (d), ECRH and NBIs injected power (e), li(3) and $\beta_{p}(f)$ and the radiated power $(g)$. 


\section{BENCHMARK AND COUPLING}

\subsection{Benchmark of FEEQS}

The direct benchmark of FEEQS has been made with the TOSCA code [18] and without METIS in the loop. TOSCA is a free-boundary equilibrium code able to evaluate the coil currents for given target parameters and the reference position of the last closed flux surface. The magnetic flux is computed with the following (generally time dependent) inputs: the plasma self-inductance, the poloidal beta (defined as $\beta_{p}=\frac{2 \mu_{0}\langle p\rangle}{\dot{B}_{p}^{2}}$, where $\langle p\rangle$ is the volume averaged plasma pressure and $\mathrm{B}_{\mathrm{p}}$ the average of the poloidal field [19]), the evolution of the plasma current, the Ejima factor [20] $\left(C_{e j i m a}=0.45\right.$ was used in this specific case [21]) and the plasma major radius. Moreover, the value of li(3) (defined as $\frac{2 V\left\langle B_{p}^{2}\right\rangle}{\left(\mu_{0} I_{p}\right)^{2} R}$ where $\mathrm{V}$ is the plasma volume and $I_{p}$ the plasma current [19]) and $\beta_{p}$ for the plasma have been chosen consistently with the expected spatial profiles of current density and plasma pressure [21]. The FEEQS code has been run in inverse mode without current in the passive structures. Also, parameters for $\mathrm{P}^{\prime}$ and $\mathrm{FF}^{\prime}$ profiles have been set to match $\beta_{p}$ and $l_{i}$ as well as possible (where $P^{\prime}$ and $F^{\prime}$ refer to the derivatives with respect to $\psi$ of the kinetic pressure $\mathrm{P}(\psi)$ and the diamagnetic function $\mathrm{F}(\psi), \nabla \mathrm{P}=\mathrm{P}^{\prime}(\Psi) \nabla \Psi$ and $\left.\nabla F=F^{\prime}(\Psi) \nabla \Psi\right)$.

The benchmark process consists of setting the CS2 coil current to the values taken from TOSCA calculations and computing all of the other currents using FEEQS. The results are reported in Figure 3(a). Also the time behaviors of the $q_{95}$ (safety factor at $95 \%$ of the poloidal flux) and the elongation computed by FEEQS are compared with the ones used in TOSCA simulations. The comparison of these two plasma parameters is reported in figure $3(\mathrm{~b})$ and in figure $3(\mathrm{c})$.
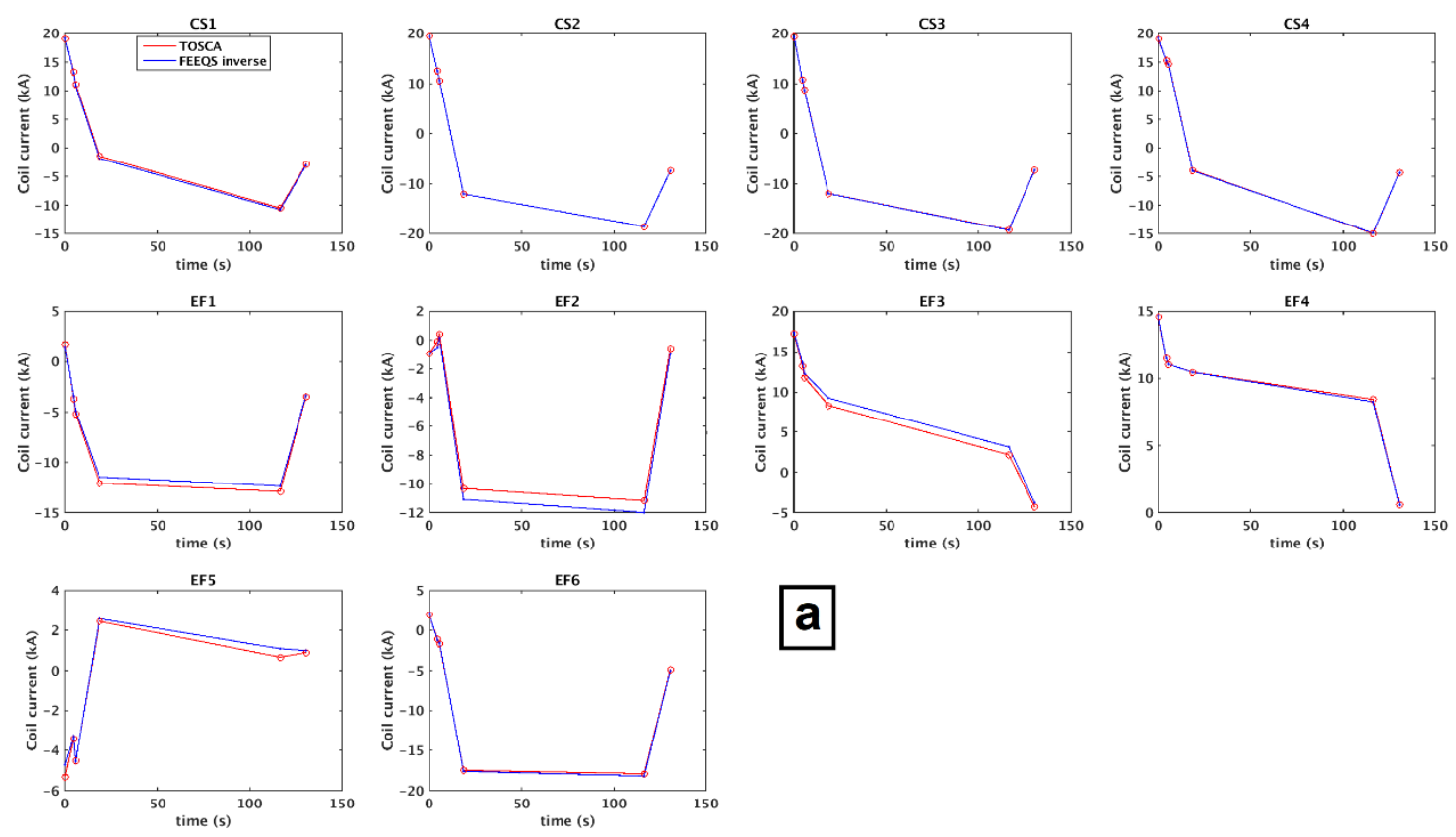

a 

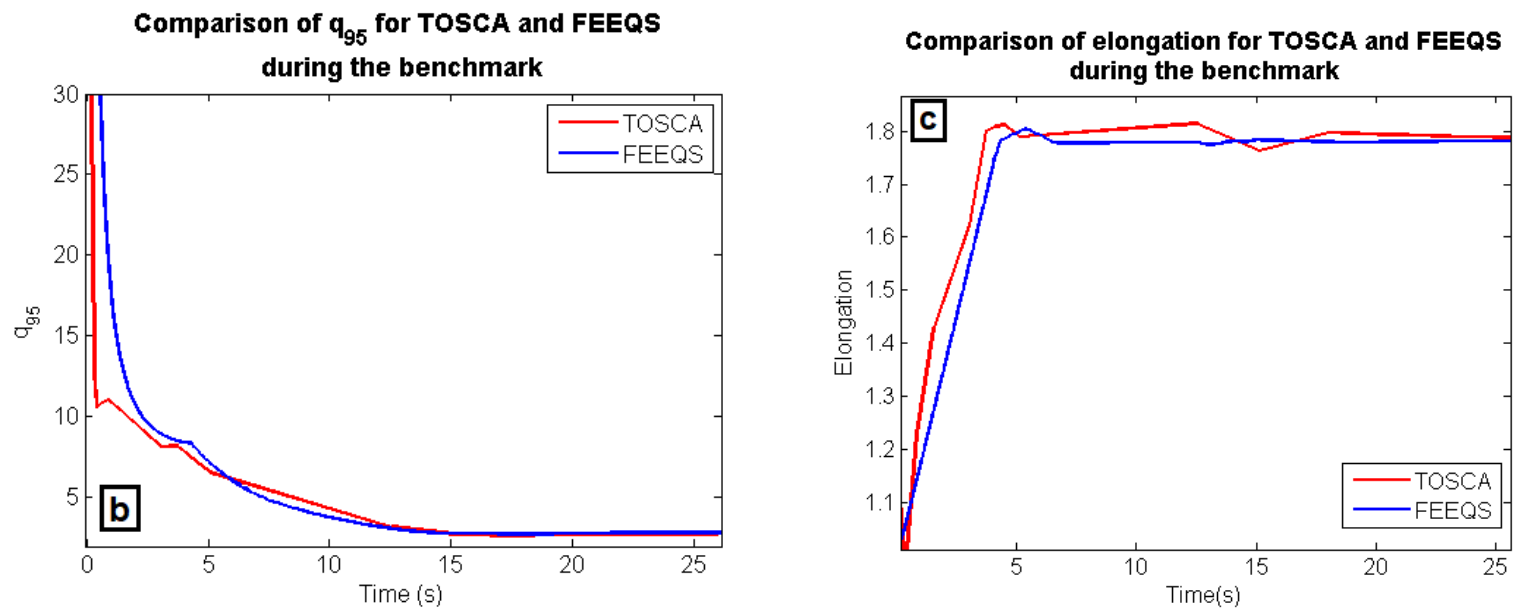

Figure 3- (a) Comparison between coil currents from FEEQS and TOSCA for the whole discharges. CS2 current is set to the values taken from TOSCA and all of the other currents are computed using FEEQS. (b) Comparison between q $_{95}$ from FEEQS and TOSCA for the first $25 \mathrm{~s}$ of the discharge. (c) Comparison between elongation from FEEQS and TOSCA for the first $25 \mathrm{~s}$ of the discharge.

As it can be seen, FEEQS results match TOSCA data well; the small differences between the two are related to the currents in passive structures, not computed in this FEEQS simulation. In fact differences appear during the ramp-up phase, related to some Ohmic flux dissipation in these structures. After the ramp-up, differences stay constant up to the end of the flat-top. Residual differences can also be due to numerical differences between the codes (such as mesh size or cost function for inverse computation).

\subsection{Coupling of METIS and FEEQS}

After this satisfactory benchmark, METIS and FEEQS have been coupled and tested, using the workflow sketched in Figure 4. The main aim of combining the two codes is to assess the effect of calculating the time evolution of $\beta_{p}$ and li(3) from the plasma profiles instead of just assuming them. Therefore, results of simulations with the coupled codes will be compared to the usual free-boundary simulations with given $\beta_{p}(t)$ and li(3)(t). In particular, similar values of the coils currents should be obtained or, in any case, within the limits given by the hardware properties.

The input data used for the benchmark and in the following analysis correspond to scenario \#2 of the JT-60SA tokamak [11]. This scenario requires a full pre-magnetization of the central solenoid (CS) coils [21], which enables to operate in a long inductive discharge at high plasma current $\left(I_{p}\right)$ of $5.5 \mathrm{MA}$. Moreover, an external heating power up to $41 \mathrm{MW}$ is provided using an ECRH system (7MW) and two NBI systems (respectively $24 \mathrm{MW}$ and $10 \mathrm{MW}$ ) [11]. Both systems contribute also to generate a fraction of the plasma current non-inductively and via the bootstrap effect.

In this way, to perform a whole discharge simulation, a starting scenario with the main parameters of scenario \#2 is generated in METIS. To prepare it, the inputs for plasma current (Figure 5(a)), line-averaged electron density, toroidal field, plasma composition, powers injected in the plasma, energy confinement multiplication $\mathrm{H}$ factor and plasma geometry are given as waveforms in time. It is possible to modify other parameters using the METIS parameters section list, such as the models for core and pedestal confinement, for sawteeth, for ITB threshold and for MHD beta limit, etc. [7]

Once the input parameters have been set, the METIS simulation is first run. From results of this simulation, the $\mathrm{P}^{\prime}$, the FF' profiles, the plasma currents, the LCFS poloidal flux and plasma shape are produced as well as the li(3) and $\beta_{p}$ profiles (Figure 5(b)) and the the flux consumption (Figure 5(c)). The second step consists in using these data together with the coils limits as constraints in FEEQS, which is 
then run to compute the coils currents, the radial hoop forces and vertical forces on the coils, as well as the corresponding applied voltages.

FEEQS then returns the LCFS of the solution of the free-boundary equilibrium problem. Then, the third step consists in updating the new LCFS in METIS and run it again. At last, the new METIS results are used to run FEEQS and to re-compute the coils currents (see Figure 4 for the complete workflow). Note that the initial LFCS shape is user-designed and is not necessarily a solution of the equilibrium equation; using this iterative procedure, a LCFS close to the initial one is obtained as a solution of the free-boundary equilibrium equation.

The coupling procedure described in the previous sections could be iterated several times, leading to smaller and smaller increments (or changes, or variations). In the following simulations the number of iterations is restricted to two. This was considered as sufficient, as a third iteration led to an average coil current variation of less than $2 \%$.

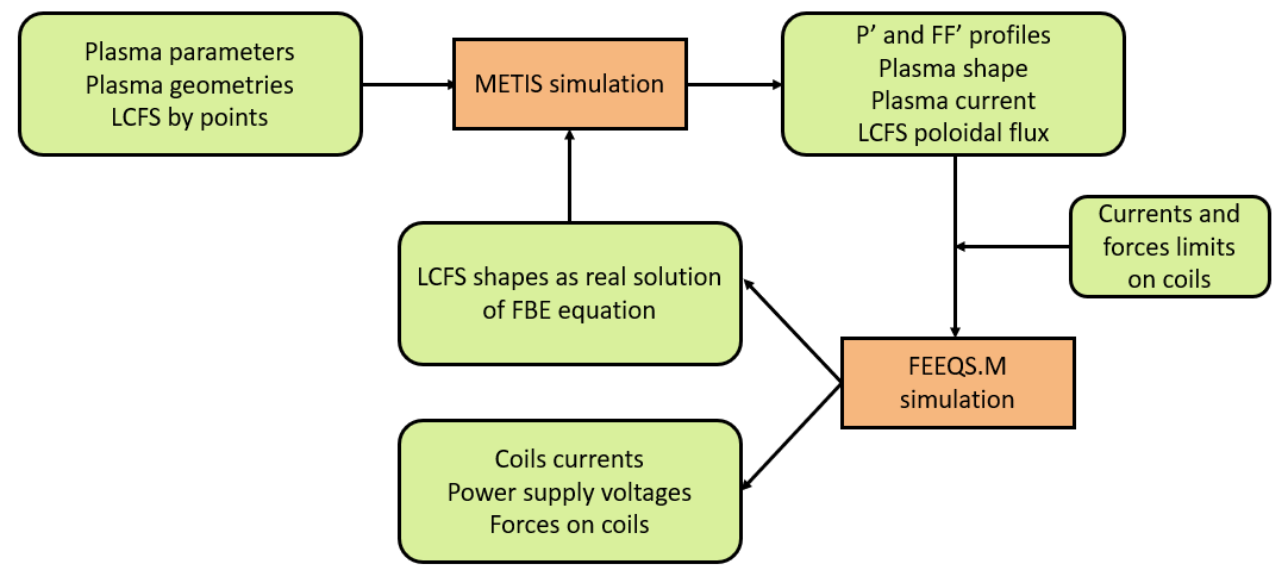

Figure 4 - Workflow combining METIS and FEEQS. In the case of the present simulations, METIS is run twice. In the first run, the LCFS is given by points; in the second run, the LCFS is computed and the full solution of the free-boundary equilibrium equation obtained by FEEQS is used.

In these simulations, the waveform of the plasma current given in Ref. [21] is used (see Figure 5(a)). In the first step, the variation of the input METIS parameters are made, in order to obtain similar behaviors of $\Psi_{\mathrm{p}}, \mathrm{q}_{95}, \mathrm{l}_{\mathrm{i}}(3)$ and $\beta_{\mathrm{p}}$ and a similar value of the $\mathrm{C}_{\mathrm{Ej} \text { jima }}$ factor used as inputs to perform the benchmark. In addition, the flux consumption is computed and compared with reference results, as it is connected to the coil currents behaviors. Figure $5(b)$ shows the comparison of li( 3 ) and $\beta_{p}$ profiles computed by METIS with respect to the time behaviors fixed for the benchmark. It is important to achieve a similar time evolution of the sum of li/ 2 and $\beta_{p}$, which is proportional to the Shafranov shift, an essential parameter of the magnetic equilibrium. It appears that all of the curves display non-negligible differences, which already shows the impact of including a plasma model in the loop. 

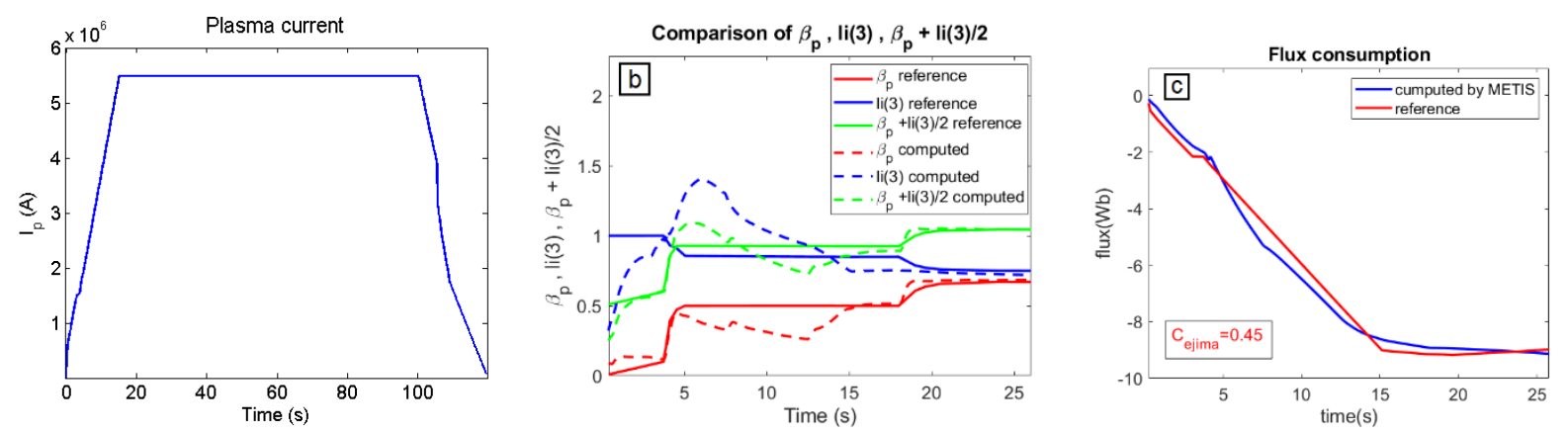

Figure 5 -First step of the simulation. (a) Plasma current time behavior taken from Ref. [21] and used as input parameter in METIS. (b) li(3), $\beta_{p}$ and li $2+\beta_{p}$ profiles computed by METIS compared with the same quantities of the simulations of Ref. [21] (used for the FEEQS-TOSCA benchmark and labelled as "reference"). (c) Comparison between the flux consumption computed by METIS and the reference one.

The Figure 5(c) shows the comparison of the flux consumption calculated by METIS and the one in Ref. [21]: their time evolutions in all the current ramp-up phase are very similar. All the comparisons take into account only the ramp-up phase since during the flat-top phase all the quantities keep the same values. Moreover, with these parameters the $C_{\mathrm{Ejjma}}$ factor is equal to 0.45 , the value used in the benchmark.

The second step consists of using the METIS results as input for FEEQS to compute coils currents and compare them to the reference ones. Although a simple model for the plasma breakdown phase exists in METIS, an accurate and reliable computation of the flux consumption during that phase is challenging [22] and affected by large uncertainties. Therefore, an offset value is added to the poloidal flux at the LCFS in order to start the simulation with initial conditions matching the reference.

The inverse method used in FEEQS to compute the coils currents is an ill-posed problem. This means that the problem has more than one solution and the same plasma equilibrium can be obtained with different sets of coils currents. For a converged solution, a regularization term is included in the model [8]. This regularization improves the conditioning of the problem, thus enabling a numerical solution. In FEEQS, the regularization term is used to select the current value closer to the one of the previous time slice. At the beginning of the simulation, FEEQS searches for a time slice of the scenario where it manages to converge providing the initial equilibrium. Afterwards, it estimates the best regularization weight, using an L-curve like algorithm [23], by computing the initial equilibrium scanning weights in the range provided as input by the user.

It is also possible to include as additional constraints the forces on coils and the forces between coils. Furthermore, a penalization of the EF5 coil current has been added to enforce the use of EF5 coil only for adjustment of the strike point position and prevent it to be used for the x-point formation; otherwise, a coil current saturation and dipole configuration with currents of opposite signs in adjacent coils occur. A penalization means that the variable penalized has a minor contribution in solving the problem but it is still incorporated in the model.

As the poloidal flux is defined with a constant offset, matching METIS and FEEQS poloidal flux require the definition of this constant. The METIS simulation starts with a null flux at the LCFS; in FEEQS the flux is defined to be zero when all the currents are zero in the entire meshed domain. This simulation is performed setting the flux offset to $17.4 \mathrm{~Wb}$; this value allows a good match of poloidal flux between METIS and FEEQS at the end of the breakdown phase. The regularization weight is set to $10^{-17}$ and the penalization of EF5 is set to 1000 (that correspond to a weight of $10^{-14}$ ). The time behavior of coils currents 
computed by the coupled codes, compared to the ones computed for the benchmark (i.e., without METIS) are shown in Figure 6. Note that the coils positions are identified in Figure 2(a).
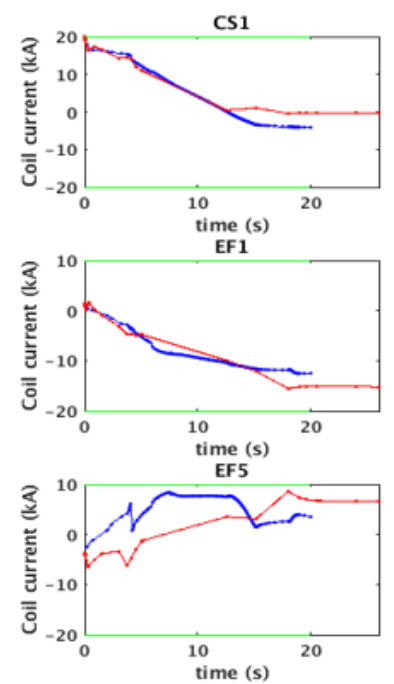

CS2

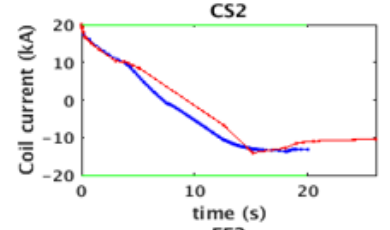

EF2
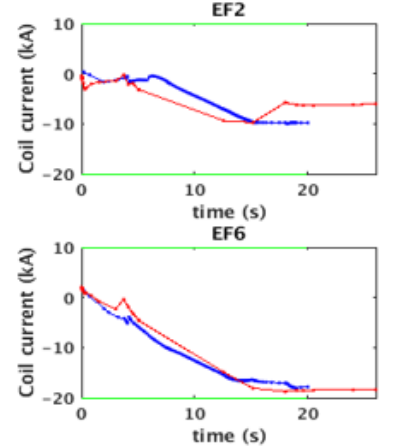

c53

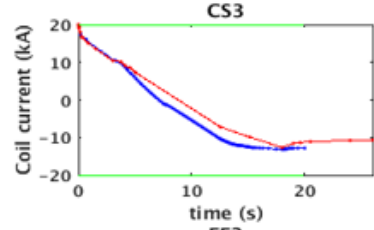

EF3

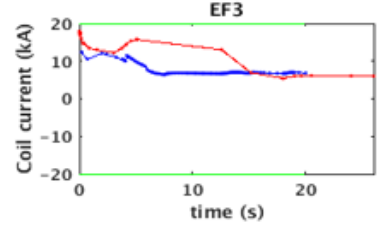

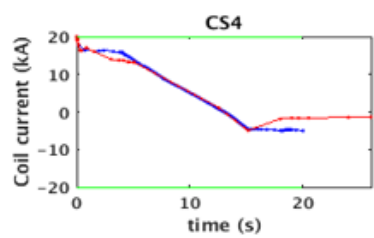

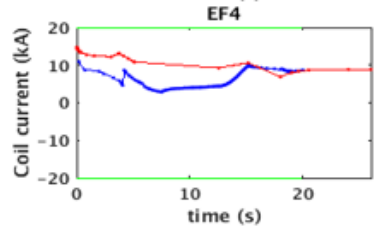

Figure 6 - Second step of the simulation. Red lines: coils currents computed by FEEQS without METIS in the loop for the rampup phase. Blue lines: coils currents computed by FEEQS coupled with METIS.

The central solenoid and the equilibrium field coils EF1 and EF6 are very similar, while EF from 2 to 5 have some differences, related to differences in the plasma shape and in $\mathrm{P}^{\prime}$ and in $\mathrm{FF}^{\prime}$ plasma profiles. Moreover, being the solution not unique, the cost function can affect the solution in slightly different ways; this can explain some differences in the results.

Finally, the impact of the simulation initial conditions has been analyzed, in particular that of the initial LCFS. To run METIS for the first time, a LCFS given by points is used. Once FEEQS is run, the LCFS solution of the free-boundary equilibrium problem is available. In FEEQS, METIS LCFS points are just a constraint used in the cost function. Therefore, the equilibrium computed by FEEQS can have a slightly different LCFS. Hence, it is possible to update the new LCFS in METIS and run the code again. $\mathrm{l}_{i}(3), \beta_{p}$ and elongation are computed and compared. The new outputs are used to run FEEQS and to compute the coils currents. The flux offset and the regularization weight are still set to $17.4 \mathrm{~Wb}$ and to $10^{-17}$ respectively.

It is found that the new time behaviors of coils currents are very similar to those computed with the LCFS given by points. Some differences on plasma quantities appear only in the very first seconds of the evolution, then the memory of the initial LCFS is lost and the evolution continues in the same way. In Figure 7(a) the differences in behaviors of $\beta_{p}$ and li(3) are plotted, in Figure 7(b) the comparison between the $q_{95}$ computed with and without the FEEQS LCFS uploaded in METIS are shown and in Figure 7(c) the two computed current density profiles at time=3.582 s are reported. Figure $7(\mathrm{~d})$ shows the difference in the LCFS given by points (dashed line) and that computed by FEEQS (solid line). 


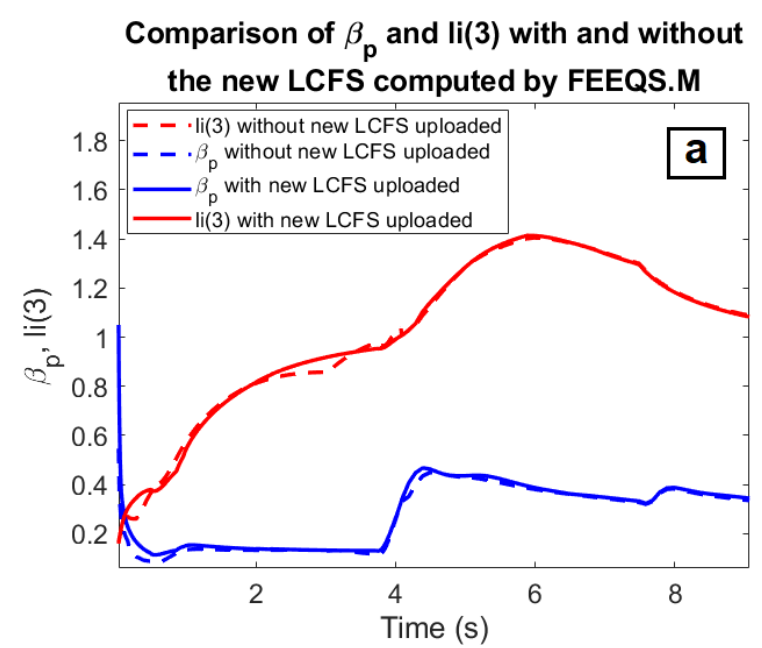

Comparison of total density profile without and

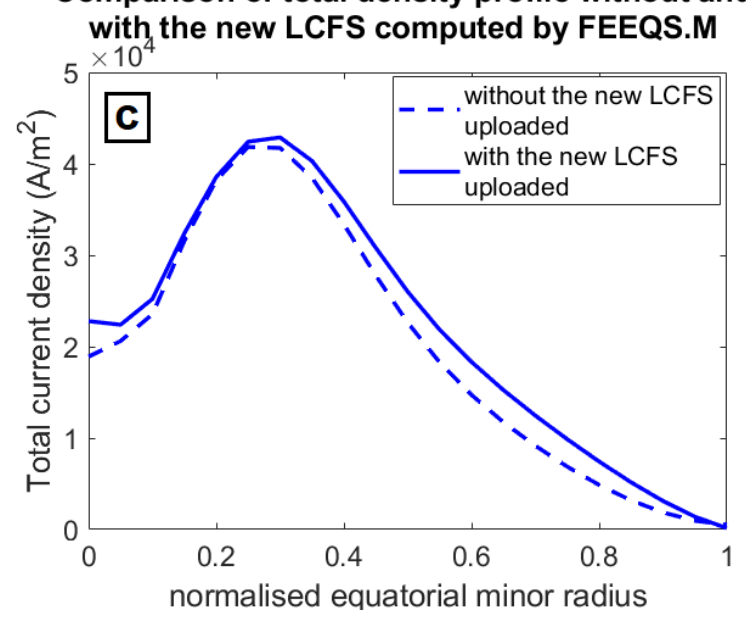

Comparison of $q 95$ and $q_{\min }$ with and without the new LCFS computed by FEEQS.M

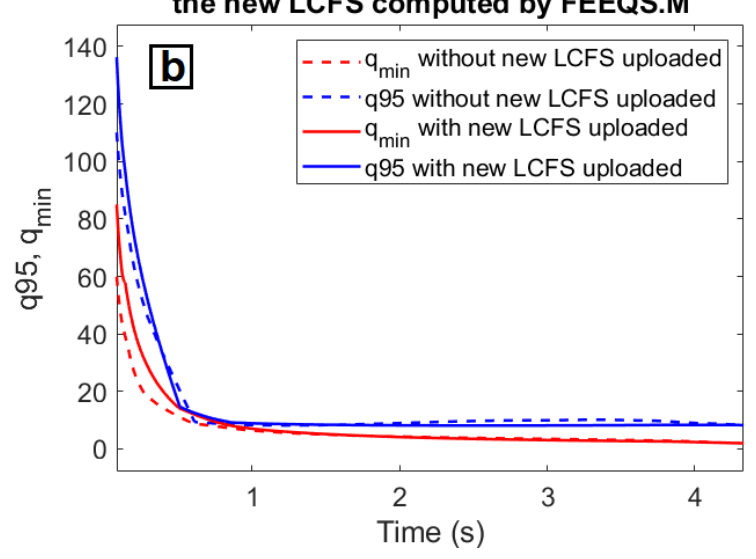

Comparison of equilibrium with and without the new LCFS computed by FEEQS.M

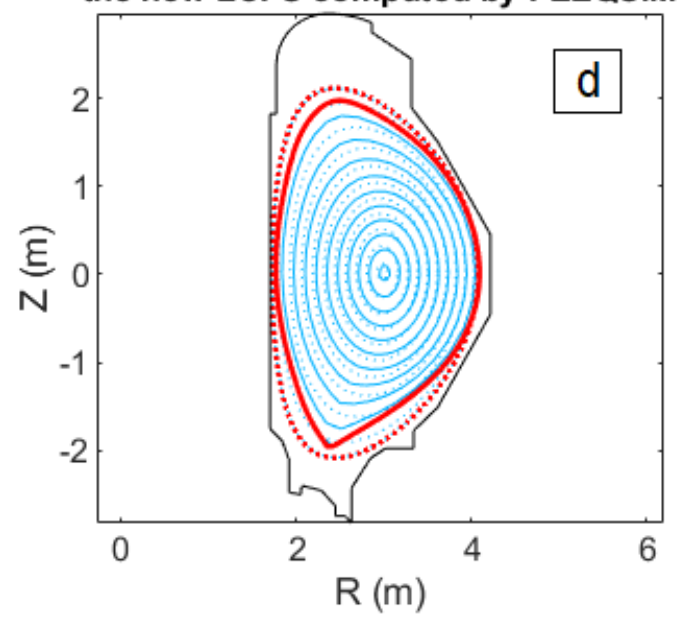

Figure 7 - Third step of simulation. (a) Comparison between $\beta_{p}$ and li(3) calculated with and without the uploaded LCFS computed by FEEQS as solution of the free-boundary equilibrium problem. (b) $q_{95}$ and $q_{\min }$ comparison between first calculation and uploaded LCFS calculation. (c) differences in behaviors of the current density profiles computed at time=3.582 s by METIS in both cases. The abscissa is the normalized Lao coordinate (normalized equatorial minor radius) [14]. (d) Comparison between equilibria with the LCFS given in METIS by points and the one computed by FEEQS at time=3.582.

Figure 7 clearly shows that the behaviors of all the quantities are quite similar after 5 seconds from the start of the discharge. The difference in the values at the very beginning are due to the different plasma shape parameters. Moreover, as it possible to notice in Figure 7, it is useful to add the new LCFS in the loop, in order to optimize the transition to a diverted configuration.

\section{APPLICATION TO SCENARIO OPTIMIZATION}

A scenario is a set of coherent, machine-independent and reproducible plasma properties. Scenario optimization means to check if the main reference parameters expected are achievable and by which experimental set-up. The process consists of launching a number of simulations and varying a set of parameters and waveforms until the scenario requirements are met. 
In METIS, using the function "scenario generator" [7], it is possible to initially define the scenario main parameters, setting the LCFS parametrization, the main gas species, the value of the plasma current, the vacuum magnetic field, the line-averaged electron density in the flat-top phase, the confinement time multiplication factor during the $\mathrm{H}$-mode phase, the maximum values of external heating power injected and the desired flat-top duration. Once these parameters have been chosen, the scenario is generated by the code.

From the METIS outputs, it is possible to check whether the available flux after breakdown is sufficient to sustain the plasma current in the whole plasma discharge. Other parameters that have been examined are the loop voltage (to avoid possible excessively large peaks), the line-averaged electron density (higher than the threshold for runaway electron formation and lower than the Greenwald limit) and the possible disruption occurrences. In the specific case of METIS, a disruption flag is generated when the radiative power exceeds the input power. Control of disruptions should be taken into account for safety in ITER, in which only a limited number of disruptions will be tolerable and because in principle no disruptions are acceptable for DEMO.

From the FEEQS outputs, the coils currents and the forces on coils are computed in order to check whether they are inside the limits related to hardware capability and safety.

Scenario optimisation depends on the careful choice of a number of input parameters and waveforms, as in the actual experiments. The advantage of the fast coupled codes we describe in the paper is that the time of each iteration in the optimisation process is very short and one can systematically check the compatibility of the scenario with the limits of the coil system. In general METIS takes 1-2 minutes per simulation done on 1000 time slices, corresponding to $0.1 s$ time steps. In FEEQS, the number of simulated time slices starts from a minimum of 11 points onwards. A simulation on 11 points takes 3-5 minutes. In general, the simulation time increases linearly with the number of simulated time slices. In the simulations in the paper, 101 time slices were used in FEEQS. Note that the time slices on which the FEEQS simulation is run are not evenly distributed, but are automatically more densely packed in the discharge phases with large parameter variations (ramp-up, ramp-down).

\subsection{Scenario \#2 of JT-60SA}

The scenario analyzed is a standard $\mathrm{H}$-mode similar to the ITER baseline scenario, however with an improved $\mathrm{H}$-factor. It consists of an inductive operation at $\mathrm{I}_{\mathrm{p}}=5.5 \mathrm{MA}$ with a flat top duration of $100 \mathrm{~s}$. The heating and current drive systems will provide neutral beam injection of 34MW $(10 \mathrm{MW}, 500 \mathrm{KeV} \mathrm{N}-\mathrm{NBI}$ and $24 \mathrm{MW}, 85 \mathrm{KeV} \mathrm{P}-\mathrm{NBI}$ ) and ECRH of $7 \mathrm{MW}$ with $138 \mathrm{GHz}$ frequency (the appropriate one for a $2.25 \mathrm{~T}$ magnetic field). The divertor target is supposed to be actively cooled in order to handle the expected heat flux up to $15 \mathrm{MW} / \mathrm{m}^{2}$ for the flat-top duration. The other main parameters of scenario \#2 are reported in Table 1. [11] [24]

Table 1 - Main parameters of scenario \#2.

\begin{tabular}{|l|l|}
\hline Major radius $(\mathrm{R})$ & $2.96(\mathrm{~m})$ \\
\hline Minor radius (a) & $1.18(\mathrm{~m})$ \\
\hline Volumetric averaged electron temperature & $6.3(\mathrm{KeV})$ \\
\hline Central averaged temperature & $13.5(\mathrm{KeV})$ \\
\hline Volume-averaged electron density & $0.56\left(10^{20} / \mathrm{m}^{3}\right)$ \\
\hline Line-averaged electron density & $0.63\left(10^{20} / \mathrm{m}^{3}\right)$ \\
\hline
\end{tabular}




\begin{tabular}{|l|l|}
\hline Central averaged electron density & $0.77\left(10^{20} / \mathrm{m}^{3}\right)$ \\
\hline $\mathrm{H}$ factor & 1.3 \\
\hline Gas & Deuterium \\
\hline
\end{tabular}

\subsection{Optimization process}

In order to optimize the considered JT-60SA scenario, the METIS simplified breakdown model for the plasma initiation is used, while ITB formation, recycling and runaway effects are not used. Matthews scaling law is used to model the line radiative power in the core plasma and the LCFS electron temperature and density are computed using confinement scaling laws. All of the simulations have been made applying a low voltage $(\mp 1 \mathrm{kV})$ to CSs, EF3 and EF4 coils during the burn-through phase, in order to reduce the values of the two voltage peaks formed during the $\mathrm{L}-\mathrm{H}$ and $\mathrm{H}-\mathrm{L}$ transition. In addition, a rapid change in additional injected power could contribute to the peaking voltages formation. A slower power ramp could be a reasonable solution for the mitigation of these peaks. The value of power for assisted breakdown and burn-through is set to $0.1 \mathrm{MW}$. The maximum power at the end of the ramp-up is set to $6.7 \mathrm{MW}$ because this value decreases the poloidal resistive flux consumption during ramp-up and allows a longer flat-top.

The waveform of the plasma current is the same used in Ref. [21] (see Figure 5(a)); the value of the plasma edge electron density is $1.84 \times 10^{19} \mathrm{~m}^{-3}$ and the value of the density peaking factor (ratio of the central to the volume-averaged density) is fixed and set to 1.3 .

The waveforms of external heating power injection and their position with respect to the centre of the plasma play a fundamental role in the optimization process. The waveform of the ECRH power injection (see Figure 8(a)) is adjusted in order to avoid disruption of the plasma connected to the x-point formation (at $4.09 \mathrm{~s}$ ). The ECRH radial deposition location is 0.5 (in normalized radius) with a Gaussian width of 0.2 ; this leads to the values of volume-averaged and central electron temperature of Table 1 (higher is the value of radial deposition position and lower is the central electron temperature, as shown in Figure 1). The P-NBI is used to assist the transition to $\mathrm{H}$-mode (at $12.89 \mathrm{~s}$ ). The waveforms of $\mathrm{N}-\mathrm{NBI}$ and $\mathrm{P}-\mathrm{NBI}$ power injections are plotted in the Figure $8(\mathrm{~b})$.
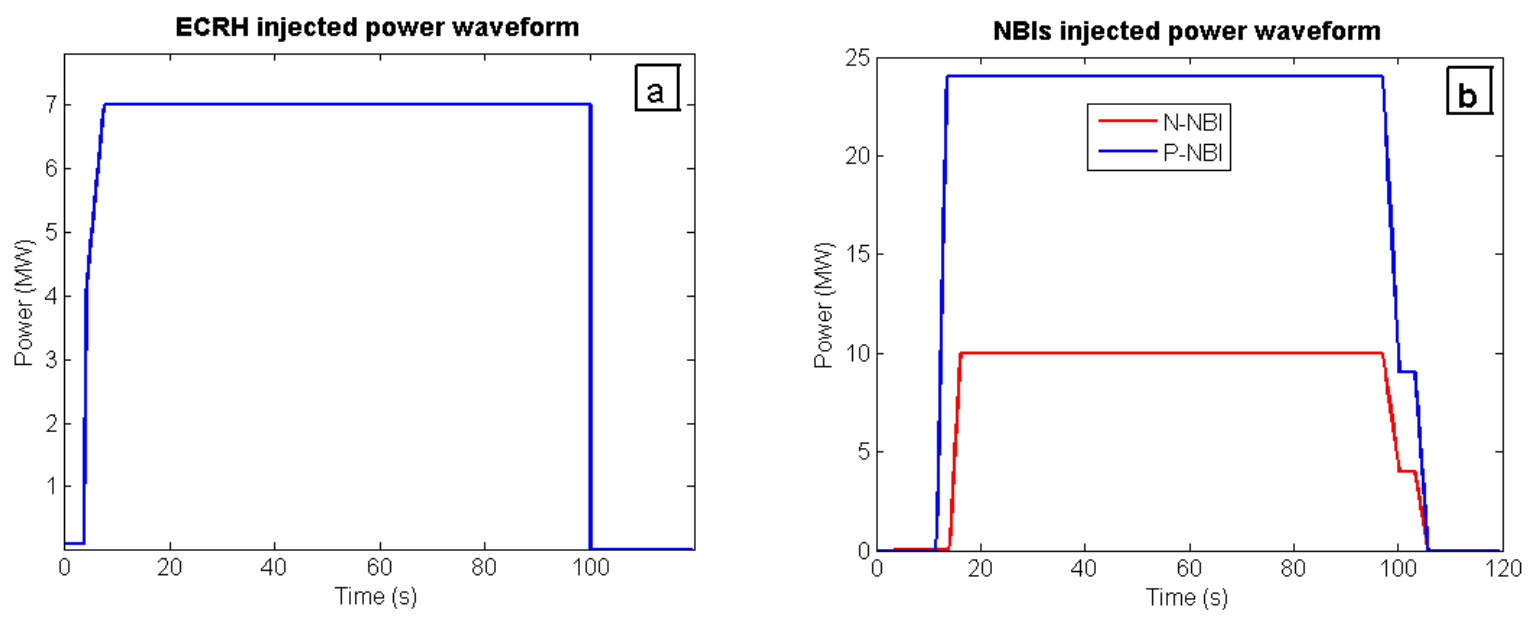

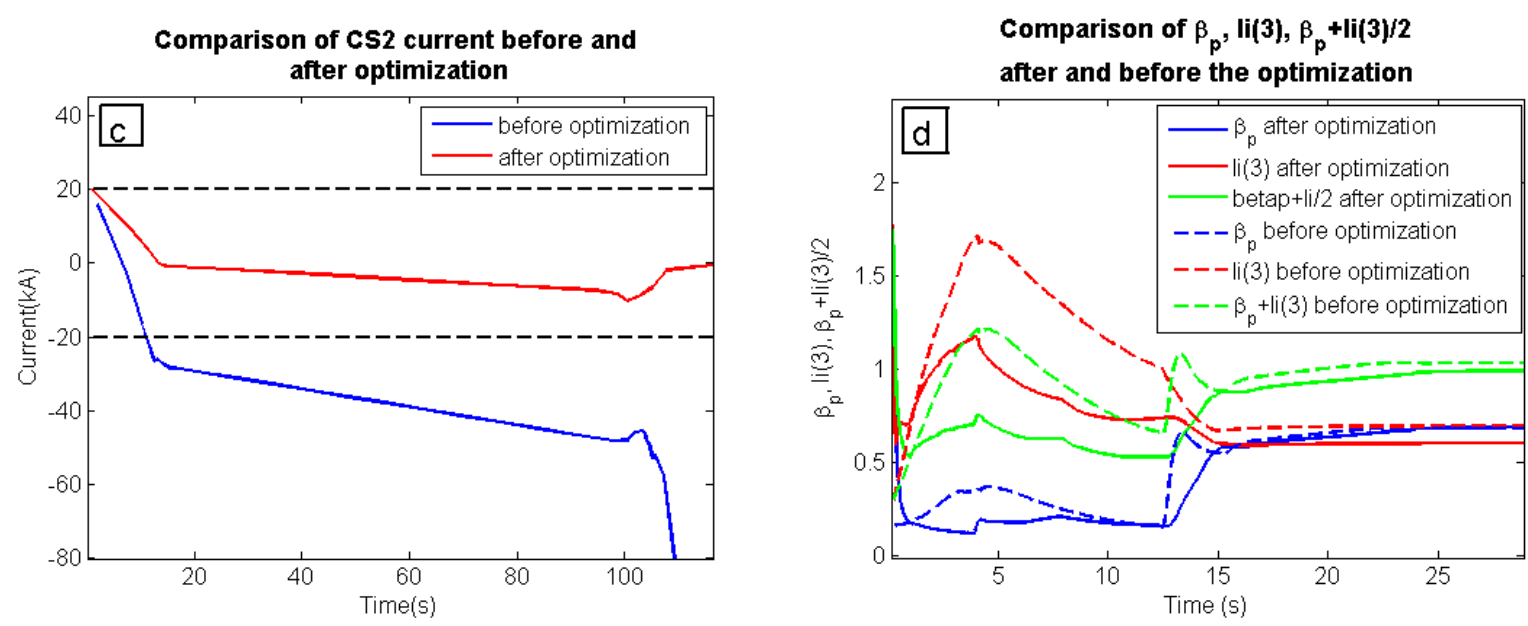

Figure 8 - (a) The waveform of the ECRH power adjusted in order to avoid the first disruption of the plasma connected to the $x$ point formation (at $4.09 \mathrm{~s}$ ). (b) The waveforms of N-NBI and P-NBI powers adjusted in order to assist the H-mode transition (12.89 s). (c) The CS2 current computed for the non-optimized case and after optimization. The dashed-lines correspond to the technological coils limit. (d) $\beta_{p}$, li(3) and $\beta_{p}+l i(3) / 2$ computed after and before the optimization.

The coils currents are computed using FEEQS before and after the optimization process, then the new LCFSs are uploaded in METIS and both codes are run again. In all of the simulations, the poloidal flux offset is set to $20 \mathrm{~Wb}$ and the regulation weight is $10^{-14}$ respectively, with the value of penalization for EF5 set to 100 . The constraint forces on coils and the constraint forces between coils are taken into account in the simulation. Figure 8(c) shows the coil currents for CS2 computed for the two cases. As it is possible to notice, for the non-optimized case the current exceeds the lower coil limit, while after the optimization the current in the same coil is within the limits. Early injection of EC waves is the main tool for reducing the flux consumption.

In figure $8(d)$ the $\beta_{p}, l i(3)$ and $\beta_{p}+l i(3) / 2$ computed after and before the optimization are shown. A large difference appears especially in the ramp-up phase: this is because the shape of the plasma is different as well as the formation of the $\mathrm{x}$-point and the $\mathrm{L}-\mathrm{H}$ transition that take place at different times in the two simulations.

At the end of the flat-top, the NBI heatings are turned off in two steps at slightly different times, as shown in figure 8 (b) in order to avoid exceeding the limits of the coil currents and voltages during the $\mathrm{H}$ L transition.

FEEQS is able to compute the radial hoop forces, the vertical forces and the voltages applied on each coil. In the optimization process, also these quantities have to stay within the imposed limits, otherwise the scenario is not feasible. Figure 9 shows the time behavior of the voltages applied on each coil and their upper and lower limit values. Peaks and oscillations, at $t=12.89 \mathrm{~s}$ and $\mathrm{t}=100 \mathrm{~s}$, correspond to the L$\mathrm{H}$ mode and to the $\mathrm{H}-\mathrm{L}$ mode transitions, as well as to the sharp increase and decrease of the power: they could be possibly controlled by a feedback system. Globally, they stay rather well within the limits, with the exception of some singular points. Note that in these simulations the LCFS is a constraint, since there is no feedback control of the shape. The large oscillations observed are mostly due to this constraint and would be relaxed using feedback shape control (e.g., through gaps control). 

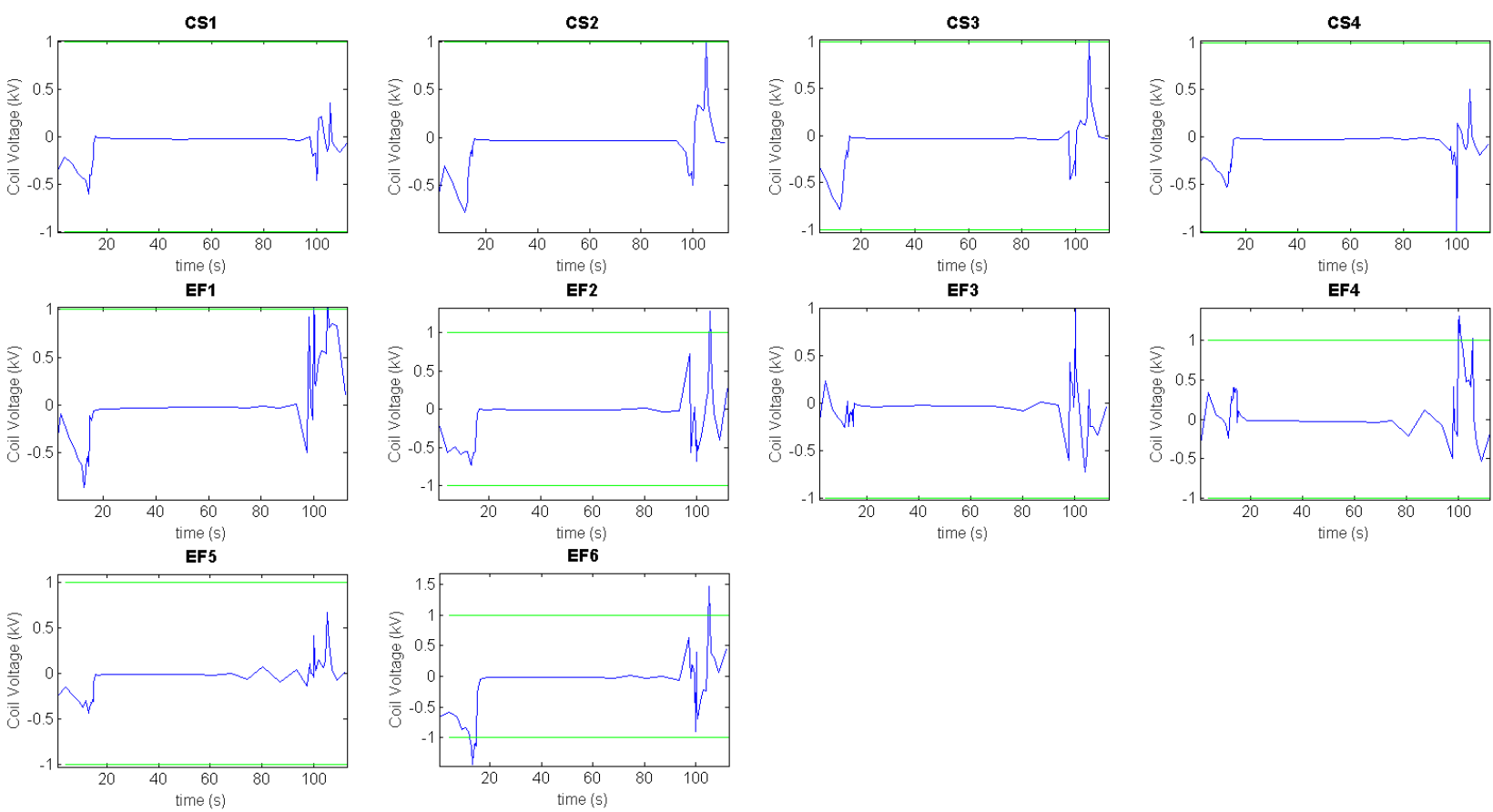

Figure 9 - coil voltage computed by FEEQS. The green lines represent the upper and the lower limits on each coil voltage. The first peaks are due to the $\mathrm{H}$-mode transition, while the second peaks are due to the loss of the $\mathrm{H}$-mode. During the flat-top phase all the voltages applied on the coils lie between the limits. Some singular points marginally exceed the limits.

In the end, the volume-averaged and the central temperatures and electron density are checked in order to verify if they are close to the typical values reported in Table 1 for the scenario and, therefore, if the scenario fulfils the requirements and is well optimized.

Since all of the plasma parameters and properties are very close to the reference values established for the scenario \#2 of JT-60SA, it is possible to conclude that the scenario meets the physics requirements and is well optimized up to the beginning of the ramp-down phase (see Figure 10). More efforts are needed to analyze the last phase of plasma in which the simulation becomes very sensitive to every change in parameters, in particular the implementation of adequate controllers. A possible future improvement is using FEEQS in inverse evolution mode [8]. In this way a self-consistent computation of the currents in the passive structures can be achieved and, therefore, a better description of transient phases.

The optimization process is also useful to highlight which are the parameters that have the largest influence on the plasma and the currents behavior. It was found that the EC waves are useful to assist the $x$-point formation during a short temporary current flat-top, in order to compensate the transient decrease of the Ohmic power. Moreover, the NBI power is necessary to assist the H-mode transition; the other fundamental parameters are the radial positions at which the various heating powers are deposited. Another parameter that can be adjusted to prevent plasma disruptions is the time evolution of the lineaveraged electron density: an increase of density leads to a decrease of temperature, at constant heating power. 

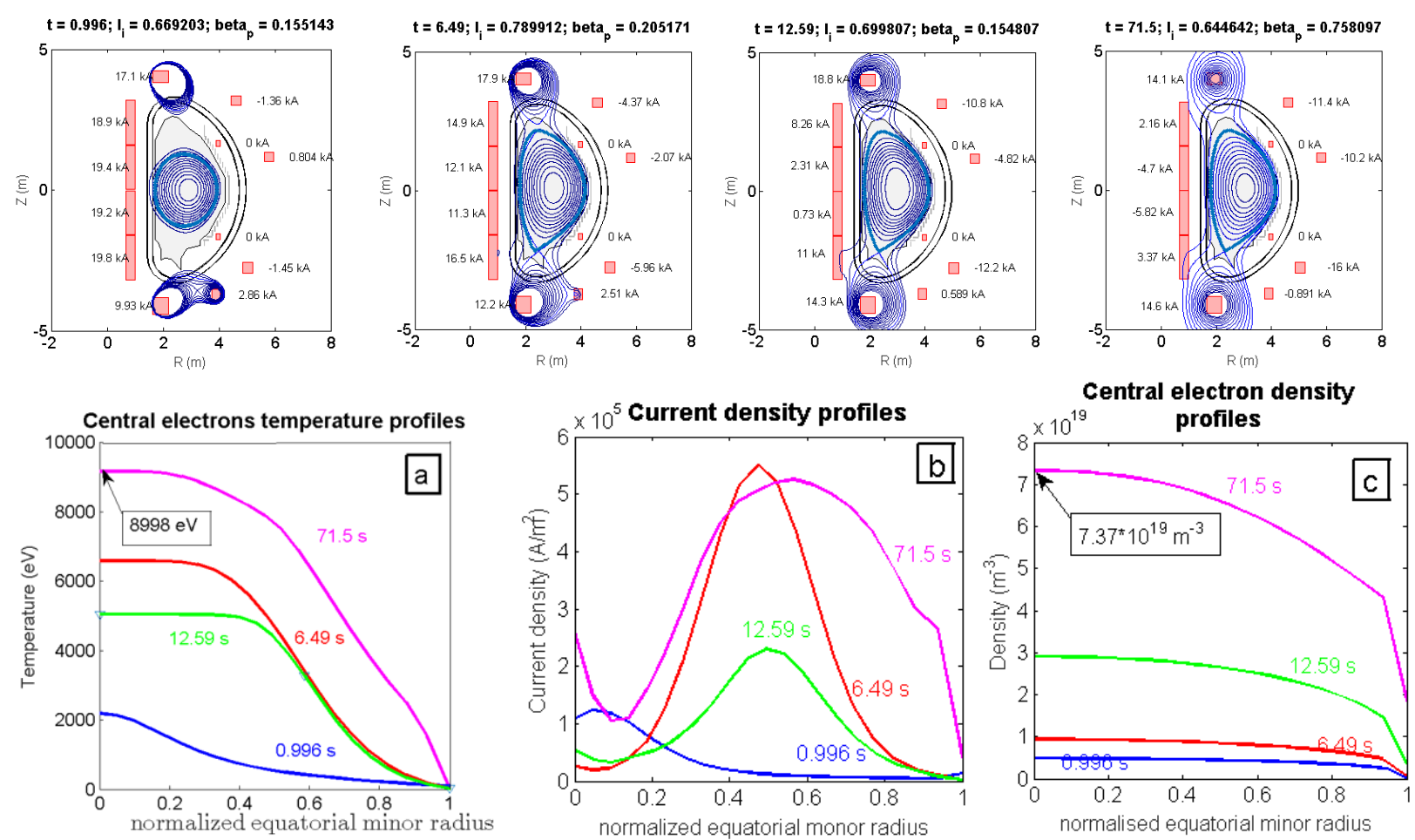

Figure 10 - Top: four plasma equilibria computed by FEEQS. From left to right the equilibrium corresponds to the time just before the $\mathrm{x}$-point formation, the time after the $\mathrm{x}$-point formation, the $\mathrm{H}$-mode transition and a generic time during the flat-top. Bottom: The central electron temperature (a), current density (b) and density (c) profiles corresponding to the four equilibria shown in the top panels. $x$ is the normalized Lao coordinate (normalized equatorial minor radius) [14].

\section{CONCLUSIONS AND PROSPECTS}

In this paper, we have described the properties of a new tokamak discharge fast simulation procedure, coupling the free-boundary magnetic equilibrium computation with modelling of the plasma state, including 1D source and loss terms. This development has been strongly motivated by the need for systematic and detailed scenario predictions, which will be mandatory for the next generation of tokamak experiments. After careful benchmarks, the use of the simulator has been tested and illustrated by a first application to one of the main reference scenarios of the large superconducting tokamak JT-60SA. This example has shown that the results of this type of coupled simulations can be significantly different from those of the two standalone codes, providing a sounder basis for tokamak discharge prediction and optimization.

The currents evolution of solenoid coils, and of poloidal coils EF1 and EF6 computed using FEEQS in the inverse mode were very similar to the references computed during the benchmark of FEEQS and TOSCA code. Contrariwise, the results obtained especially for the currents inside the coils devoted to the $\mathrm{x}$-point formation and position (EF5) are not precisely matching. Nevertheless, these discrepancies are due to the difference in plasma shape and in $\mathrm{P}^{\prime}$ and in FF' plasma profiles. This highlights how much the evolution of the plasma discharge is sensitive to the geometrical plasma parameters and to radial profiles of plasma quantities. Therefore, developing a coupled system of codes, such as METIS-FEEQS, is well justified and is expected to yield a much more accurate description of the plasma discharges. 
Regarding the scenario optimization, the application of the coupled codes to this process has shown successful results. First, parameters established for the scenario \#2 of JT-60SA are shown to be achievable, provided additional heatings are carefully used in the ramp-up phase. The optimization process has also identified the parameters that have the largest influence on the plasma and the coils currents behavior. Since the METIS code takes as input data a set of the controllable parameters during a real experiment, this kind of simulation helps in understanding how to design the scenario and how to control the stability of the plasma during a whole discharge.

Moreover, this type of analysis is also useful to identify the elements of the computation that need improvement. A controlled plasma is essential in order to reduce the disruption occurrence, therefore more efforts are needed to better analyze the parts of the plasma discharge that are most sensitive to changes in parameters, such as the ramp-down phase.

A not less important improvement in the code consists of finding an automatic model to match the current diffusion LCFS poloidal flux computed by METIS with the LCFS poloidal flux computed by FEEQS. This improvement would lead to automatically match coils currents computed for the pre-magnetization with the coils currents computed with the METIS+FEEQS simulator.

A future step in the development of a combined simulator is to couple METIS-FEEQS and CREATE-NL [9], with its advanced plasma shape and current controllers, in order to better simulate transient phases. This additional step will allow assessment of scenario controllability, in contrast with the fast simulator approach described in this paper, which verifies scenario feasibility. Starting from a scenario that is already optimised by the fast simulator, the full simulation will be simpler to realise. Moreover, coil current waveforms provided by the fast simulator can be directly used as feedforward waveforms for the full simulator. An example of feedback control schemes that is used in tokamak operation is shape adjustment by gaps control (distance between the LCFS and the wall), which has been extensively studied for JT-60SA (in the framework of this research project) using the CREATE-NL code with the Extreme Shape Controller and documented in Ref. [25]. Later on, control strategies for kinetic profiles (as temperatures, density or safety factor) could be implemented and tested. Using as constraint the METIS prediction for plasma current, li and $\beta_{p}$, CREATE-NL provides the last closed flux surface shape to METIS for the flat-top and allows closed-loop simulations. This coupling will improve the simulation of a whole tokamak discharge and especially of ramp-up and ramp-down phases.

\section{Acknowledgments}

This work has been carried out within the framework of the EUROfusion Consortium and has received funding from the EURATOM research and training programme 2019-2020 under grant agreement No 633053. The views and opinions expressed herein do not necessarily reflect those of the European Commission. The authors gratefully acknowledge members of the JT-60SA Integrated Project Team for data exchange and fruitful discussions. This work has been partially funded by Politecnico di Torino.

\section{References}

[1] European Research Roadmap to the Realization of Fusion Energy, 2018. https://www.eurofusion.org/eurofusion/roadmap/

[2] V. Lukash et al., "Simulations of ITER scenarios". In: Plasma Devices and Operations 13 (2005) 143.

[3] S.H. Kim et al., "Investigation of key parameters for the development of reliable ITER baseline operation scenarios using CORSICA". In: Nucl. Fusion 58 (2018) 056013. 
[4] K. Besseghir et al., "Achieving and sustaining advanced scenarios in ITER modelled by CRONOS and DINA-CH". In: Plasma Phys. Contr. Fusion 55 (2013) 125012.

[5] E. Fable et al., "Novel free-boundary equilibrium and transport solver with theory-based models and its validation against ASDEX Upgrade current ramp scenarios". In: Plasma Phys. Contr. Fusion 55 (2013) 124028.

[6] V. Parail et al., "Self-consistent simulation of plasma scenarios for ITER using a combination of 1.5D transport codes and free-boundary equilibrium codes". In: Nucl. Fusion 53 (2013) 113002.

[7] J.F. Artaud et al., "Metis: a fast integrated tokamak modelling tool for scenario design". In: Nucl. Fusion 58 (2018) 105001.

[8] H.Heumann et al., "Quasistatic Free-Boundary Equilibrium of Toroidal Plasma with CEDRES++: Computational Methods and Applications". In: Journal of Plasma Physics 81 (2015) 905810301.

[9] R. Albanese et al., "CREATE-NL+: A robust control-oriented free boundary dynamic plasma equilibrium solver" Fus. Eng. Des. 96-97 (2015) 664.

[10] P. Barabaschi et al. 2019 Nucl. Fusion 59112005

[11] JT-60SA Research Plan, Sept. 2018, www.jt60sa.org/pdfs/JT-60SA Res Plan.pdf

[12] J.F. Artaud et al., "The CRONOS suite of codes for integrated tokamak modelling". In: Nucl. Fusion 50 (2010), 043001.

[13] G. Giruzzi et al, "Integrated modelling of JT-60SA scenarios with the METIS code", 39th EPS Conference \& 16th Int. Congress on Plasma Physics, P5.018, 2 - 6 July 2012. Stockholm, Sweden [14] L.L. Lao et al. "Variational moment solutions to the Grad-Shafranov equation". In: Phys. of Fluids 24 (1981) 1436

[15] R. Albanese et al., "On the solution of the magnetic flux equation in an infinite domain". In: EPS. 8th Europhysics Conf. on Computing in Plasma Physics, European Physical Society, Mulhouse, France, 1986, pp. 41-44.

[16] ] J. Blum, "Numerical simulation and optimal control in plasma physics". John Wiley and Sons Inc (USA), 1989.

[17] B. Faugeras and H. Heumann, "FEM-BEM coupling methods for tokamak plasma axisymmetric freeboundary equilibrium computations in unbounded domains". In: Journal of Computational Physics 343 (2017) 201.

[18] K. Shinya, J. Plasma Fusion Res. 76 (2000) 479.

[19] ITER Physics Guidelines, ITER report N 19. FDR 1 01-07-13 R 0.1

[20] S. Ejima et al. "Volt-second analysis and consumption in doublet iii plasmas". In: 1982 Nucl. Fusion 221313

[21] H. Urano et al. "Development of operation scenarios for plasma breakdown and current ramp-up phases in JT-60SA tokamak". In: Fusion Engineering and Design 100 (2015) 345-356.

[22] H. Urano et al. "Breakdown optimization method based on inverse reconstruction of magnetic fluxes in JT-60SA". In: Nucl. Fusion 60 (2020) 066002

[23] F. S V. Bazan et al. "An improved fixed-point algorithm for determining a Tikhonov regularization parameter". In: Inverse Problems 25 (2009) 045007

[24] L. Garzotti et al., "Analysis of JT-60SA operational scenarios". In: Nucl. Fusion 58 (2018) 026029

[25] D. Corona et al., "Plasma shape control assessment for JT-60SA using the CREATE tools". In: Fusion Eng. Des. 146 (2019) 1773. 